\title{
IMAGINAIRES DE LA MOBILITÉ CULTURELLE DANS LES ÉCRITURES (TRANS)MIGRANTES AU QUÉBEC
}

\author{
Adina Balint ${ }^{1}$
}

\begin{abstract}
Résumé: À l'heure de la mondialisation, comment repenser les imaginaires de la mobilité culturelle dans le contexte d'appartenances multiples et d'héritages pluriels ? À partir des années 1980, le phénomène littéraire des écritures dites de la migration apparaît comme un courant littéraire fascinant, notamment parce qu'il lie, de manière historique, l'évolution de la littérature québécoise aux grands courants de pensée de la fin du vingtième siècle, définis par le postmodernisme, le déplacement des individus et des populations et le transculturalisme. Des auteures nées à l'étranger et établies au Québec explorent des conditions identitaires qui tendent aujourd'hui à la multiplicité des appartenances et à la reconnaissance de l'altérité. En convoquant les textes de Régine Robin et les explorations de Pierre Ouellet, cet article examine le renouvellement de la littérature québécoise contemporaine, qui intègre les écritures (trans)migrantes et la circulation des idées et des savoirs dans une perspective d'ouverture au monde.
\end{abstract}

Mots-clés: Mobilité culturelle. Transculturalisme. Écriture migrante. Régine Robin. Québec.

\section{IMAGINANDO A MOBILIDADE CULTURAL EM ESCRITURAS QUEBEC (TRANS) MIGRANTES}

Resumo: Na era da globalização, como repensar a imaginação da mobilidade cultural no contexto de múltiplas afiliações e heranças plurais? A partir de 1980, o fenômeno literário das escrituras ditas de migração aparece como uma tendência literária fascinante, em parte porque ele liga, de forma histórica, a evolução da literatura quebequense às grandes correntes de pensamento, do final do século XX, definidas pelo pós-modernismo, com o deslocamento de indivíduos e populações e o transculturalismo. Autores nascidos no exterior e estabelecidos no Quebec exploram condições de identidade que tendem hoje à multiplicidade de pertenças e ao reconhecimento da alteridade. Ao convocar os textos de Régine Robin e as reflexões de Pierre Ouellet, este artigo analisa a renovação da literatura contemporânea do Québec, que inclui as escrituras (trans)migrantes e a circulação de idéias e de saberes, com vistas a uma perspectiva de abertura para o mundo.

Palavras-chave: Mobilidade cultural. Transculturalismo. Migrando a escrita. Regine Robin. Quebec.

\footnotetext{
${ }^{1}$ Université de Winnipeg, Canada.
} 


\section{Introduction}

Au Québec, à partir des années 1980, les écritures dites migrantes transmettent un éclatement des schémas identitaires et pensent l'altérité dans une perspective positive, où peuvent se manifester plurilinguisme et métissage. De plus en plus, on pense donc l'altérité comme «lieu de négociation» (ROBIN, 2014, web) dans les interactions culturelles pour inventer une nouvelle durée longue, orientée vers l'avenir d'un vivre ensemble, qui échappe au nationalisme enraciné valorisant uniquement le territoire où l'on est né, et cela en faveur de l'affirmation d'une citoyenneté partagée. Cette vision se manifeste, par exemple, dans le roman d'Éric Dupont intitulé La logeuse (2006). Elle se retrouve aussi dans l'ouvrage de Gérard Bouchard intitulé L'interculturalisme: un point de vue québécois (2012). Il y propose une conception de l'interculturalisme comme modèle de gestion de la diversité ethnoculturelle au Québec, dans l'esprit du pluralisme. Ce modèle est essentiellement axé sur la recherche d'équilibres entre des impératifs souvent divergents. D'une façon générale, selon Bouchard, l'interculturalisme entend tracer une voie entre des dynamiques qui tendent soit vers l'assimilation, soit vers la segmentation. Dans cet esprit, il met l'accent sur l'intégration, ce qui entraîne quatre propositions: a) œuvrer à l'insertion sociale et économique de tous les citoyens, en particulier des immigrants; b) lutter contre toutes les sources et formes de discrimination, notamment le racisme; c) promouvoir des échanges, rencontres, interactions entre individus et groupes; d) encourager la création d'une culture commune à partir et audelà de la diversité ethnoculturelle, mais sans faire obstacle à cette diversité. Dans le cas de la société québécoise, s'ajoute une composante essentielle: la promotion du français comme langue officielle en lien avec la loi 101. À cela se joint une autre singularité qui doit être prise en compte au Québec: l'existence d'une majorité francophone qui est en même temps une minorité à l'échelle du Canada et des Amériques. C'est pour cela que le multiculturalisme canadien théorisé par Will Kymlicka (Multicultural Odysseys, 2009) est remplacé au Québec par l'interculturalisme qui demande plus fortement de reconnaître des valeurs minoritaires. Ainsi, on demande aux enfants des immigrants de s'inscrire dans les écoles francophones afin de devenir francophones et non anglophones et ainsi d'accroître la population francophone plutôt que de jouer le rôle d'agent inconscient d'assimilation par l'anglophonie. 
Pourquoi cette réflexion sur un modèle de gestion de la diversité au Québec ? D'abord, on reconnaît que pour une société qui vit des changements rapides et des déplacements de populations - qui sont aussi ceux du monde contemporain mondialisé - il est utile d'avoir et de transmettre une vision du lien qu'elle entend instituer entre ses membres et des valeurs à promouvoir. De ce point de vue, le rôle joué par le multiculturalisme au Canada anglophone, par le régime républicain en France, ou par le discours du métissage dans le passé de plusieurs pays latino-américains et dans les Caraibes $^{2}$ peuvent servir d'analogies. En second lieu, - on le voit particulièrement dans la littérature - les phénomènes identitaires, la quête d'une place dans la société et sur la scène littéraire, la question de l'appropriation et de la désappropriation des objets et valeurs culturels, les effets symboliques de la migration de l'imaginaire individuel et collectif - tout cela peut constituer un lieu de division et de controverse, donc, il est bon d'explorer des orientations par des écrits littéraires. C'est ce que font les écrivains néo-québécois, migrants ou pas. Enfin, à l'heure où nombre de nations dans le monde sont interpelées par de nouvelles formes d'immigration et les défis qu'elles posent aux sociétés démocratiques, on comprend la recherche de nouveaux modèles d'intégration transculturelle appuyés sur l'idée de réseaux plutôt que sur celle de racines, comme le montre Patrick Imbert dans sa monographie Comparer le Canada et les Amériques. Des racines aux réseaux transculturels(2014).

Qu'en est-il alors dans ce contexte des écrivains néo-québécois migrants ? Ou encore quelle place y a-t-il pour les écrivains de la migration ? Y a-t-il une spécificité des écritures migrantes ? Et est-il simplement pertinent de poser ainsi le problème ? Dans son article «Écrire français avec un accent», Régine Robin écrit:

Ce qui manque à l'écriture québécoise, à la structuration du champ littéraire au Québec, c'est un Thomas Bernhard québécois. Quelqu'un qui viendrait à bout de ce défaut d'imaginaire autre par plénitude d'identité (alors même qu'elle est vécue comme fragile), plénitude d'identité qui empêche au vide de pouvoir pénétrer le jeu ou du jeu, qui empêche une réelle problématisation de prendre place (ROBIN, 2014, web).

${ }^{2}$ Voir: Joël Des Rosiers, Théories caraïbes, Montréal, Tryptique, 1996. 
On voit se dessiner une problématique qui fait de l'écrivain migrant la figure emblématique de celui qui passe au-delà des références établies et des réflexes de souche, celui qui transgresse l'imaginaire du terroir. Cet écrivain ne se reconnaît pas d'emblée dans le patrimoine national québécois, même s'il partage le même code linguistique. Il ou elle écrit, dit sa différence, raconte des histoires d'ailleurs, des histoires d'entre-deux ou de plusieurs cultures, comme le font Sergio Kokis, Ying Chen, Dany Laferrière, Nancy Huston, Kim Thúy, par exemple. Ici, c'est la mémoire qui n'arrive pas à se partager. Dans ce scénario, ce n'est pas le problème de l'accent qui est à mettre au premier plan, ni celui de la créolisation ou du métissage, mais celui de la cohérence d'un noyau langue-culture qui ne parvient pas à se défaire de son image identitaire.

\section{Singularités des écrivains de la migration ?}

Dès qu'on interroge la spécificité des écrivains de la migration, on réalise qu'on risque de tomber dans le piège des oppositions réductrices: ici/ailleurs, natif/étranger, intérieur/extérieur, durée longue/Nouveau Monde/instant ${ }^{3}$, et qu'on ne peut pas trouver des traits pertinents qui les isoleraient des autres écrivains, des autres écritures. Ils ont bien en commun d'être nés ailleurs, d'être immigrants, d'obliger les autres écrivains à s'interroger sur le partage de la mémoire, de la culture. Nous pouvons qualifier ces écritures transculturelles de plurielles et de hybrides. Qu'est-ce qu'un texte hybride?, demande Sherry Simon:

Il s'agit d'un texte qui interroge les imaginaires de l'appartenance, en faisant état de dissonances et d'interférences de diverses sortes. On peut dire que dans certains cas ces effets de dissonance sont le résultat d'un processus de traduction inachevée [...]. Le texte hybride est donc un texte qui manifeste des «effets de traduction» par un vocabulaire disparate, une syntaxe inhabituelle, un dénuement déterritorialisant, des interférences linguistiques ou culturelles, une certaine ouverture ou faiblesse sur le plan de la maîtrise linguistique ou du tissu de références. Ces effets esthétiques sont le résultat de la situation de frontière

\footnotetext{
${ }^{3}$ Voir Patrick Imbert (dir.), Rencontres multiculturelles. Imprévus et coïncidences. Le Canada et les Amériques, Ottawa, Chaire de recherche "Canada: Enjeux sociaux et culturels dans une société du savoir», 2013, ainsi que Les Amériques transculturelles. Les stéréotypes du jeu à somme nulle, Québec, Presses de l’Université Laval, 2013.
} 
que vit l'écrivain, qui par sa prise de conscience de la multiplicité choisit de créer un texte créolisé, selon l'expression d'Édouard Glissant, c'est-à-dire un texte où la confrontation des éléments disparates produit du nouveau, de l'imprévisible (SIMON, 1998, p. 233-34).

Ces écritures transculturelles sont des textes de la frontière, elles transgressent, opèrent le passage de l'identité assignée à celle de la traversée, de la mobilité. Elles mettent en scène des identités de parcours, d'itinéraires en déplacement, dans une dynamique de l'imprévisible. Ce sont des écritures de l'entre-deux, de l'interstice ou encore, de la transportation et de la transmutation. Cela implique souvent, du moins au niveau des premières œuvres, que le récit jalonne des cheminements, des souvenirs d'enfance, des nostalgies d'un pays qu'on a quitté, des comparaisons culturelles, des errances. Néanmoins, ces œuvres n'inscrivent pas uniquement la perte et une sorte de mélancolie, mais aussi des espoirs de recommencement, des promesses, des ambitions, des réincarnations avec une tension entre des désappropriations et appropriations des valeurs et autres chocs et distorsions.

Rappelons ici le premier roman de Régine Robin, La Québécoite, qui paraît en 1983, la même année que Vice Versa, et qui n'est pas un roman mais plutôt "une expérimentation à la fois littéraire et sociale» dans le but de «fictionnaliser l'inquiétante étrangeté que crée le choc culturel» (ROBIN, 1983, p. 207; 224). C'est le récit d'une arrivée en ville, à Montréal, ville cosmopolite mais qui demeure étrangère à la narratrice. Dans la même veine des chocs culturels, Le Pavillon des miroirs, premier roman de Sergio Kokis, auteur québécois d'origine brésilienne, met en lumière le tiraillement d'un narrateur désorienté, pris entre plusieurs entre-deux (temporel, spatial, d'imaginaire), où il tente de recomposer son identité:

Ce déraciné oscille ainsi entre deux temps, le sien et le réel, en arrière et en avant, sans pouvoir se fixer. C'est que le temps s'allonge drôlement, il devient élastique et visqueux à la fois, fuyant et assommant, dès qu'on s'en va de sa maison. De toute maison, ailleurs. Il passe désormais sans toutefois passer, car l'identité n'est plus en harmonie avec le monde palpable. Ses repères sont restés en arrière et lui tirent les pieds comme les fantômes d'autrefois. L'étranger ne peut pas toujours se détacher vers l'avenir; il reste souvent embourbé entre cette identité qui fut et la béance de devoir devenir autre (KOKIS, 1994, p. 357). 
Romans de la déterritorialisation, de la dissonance, de la noncoïncidence entre soi et le devenir-autre, du «mineur», du rhizome plutôt que de la racine, ces écritures dites migrantes ne sont-elles pas l'illustration la plus saisissante des transgressions connues par la littérature québécoise contemporaine? Une littérature québécoise ouverte aujourd'hui à l'internationalisation et à l'entrée en force d'écrivains nés à l'étranger dans ses rangs.

Dans son ouvrage L'esprit migrateur. Essai sur le non-sens commun, Pierre Ouellet souligne que le thème de la migration s'est largement répandu dans la littérature contemporaine, notamment depuis la multiplication des écrivains «migrants» au Québec, comme ailleurs. Cette migrance n'est pas uniquement géoculturelle, liée au déplacement d'un territoire à un autre:

[...] elle est aussi de nature ontologique et symbolique, puisqu'elle caractérise le déplacement même du Sens et de l'Etre dans l'expérience intime de l'altérité, où l'on fait preuve du non-sens ou du néant de son identité, individuelle ou collective, qui n'existe pas sans l'appel à l'autre où elle se métamorphose à chaque instant (OUELLET, 2005, p. 9).

Ainsi est-il que l'écrivain contemporain, migrant ou pas, serait l'alchimiste d'une forme de dissidence et de non-coïncidence de ce que l'on attend de lui. Il ne serait plus l'écrivain de la migration, de l'exil, ni de l'errance, mais pour emprunter un terme à Ouellet, écrivain de la «transportation, transmigration, transmutation» (2010, p. 289). Dans ces mots valise, on entend à la fois la mobilité des corps et de l'information, qui caractérise notre époque - comme lorsqu'on parle de la migration des personnes ou de la circulation des signes et des symboles -, et la mobilité qui touche aussi l'esprit ou la conscience, le souffle, l'âme, qu'on identifie souvent à une sorte de principe de vie.

Dans cette perspective, il n'y a donc pas de frontière nette entre les écrivains de la migration et les autres. Les écrivains sont aujourd'hui de nouveaux nomades de notre monde éclaté, fragmenté, inter et multiculturel, porté par un imaginaire de la multiplicité: plusieurs souffles, plusieurs langues, des allers et retours, des reconfigurations, des phases d'exil plus ou moins temporaires, des itinéraires. À cela s'ajoutent des imaginaires de la métamorphose au niveau des genres et des formes littéraires. En effet, les écritures contemporaines sont hybrides, au sens où elles sont inclassables : 
récits, romans, autofictions, documents, essais? Elles réinventent des formes non-canoniques du roman en réinscrivant dans leurs textes la chronique, le collage, le montage, l'intertexte, la citation, des jeux de langue, le bilinguisme... - ce qui nous convie évidemment à repenser nos habitudes de lecture et d'interprétation, à transgresser des frontières, à lire non plus le totalisant ou l'homogène mais le fragmentaire.

\section{«Nous autres»: visages de l'altérité}

Nous autres, vous autres, eux autres... Parler de ce qu'on est, d'où on vient, du groupe auquel on appartient au Québec passe par l'utilisation du mot «autre». Il s'agit d'inscrire constamment une différence en employant le pluriel: «nous», "vous», «eux», presque jamais «moi autre». La formule «nous autres» marque ainsi une altérité entretenue, choisie, entrée dans l'usage; c'est dire l'appartenance à un groupe défini autour d'un «nous» identitaire ${ }^{4}$, alors que "vous autres», "eux autres» sont ceux qui ne sont pas comme «nous». On voit surgir ici plusieurs questions qui se déploient dans le roman La Québécoite de Régine Robin: la question de l'identité et de l'altérité (celui qui est né ici et celui qui vient d'ailleurs), l'inclusion-exclusion, le groupe dominant et les autres... Quelle que soit l'interrogation autour du dualisme, elle demande à être nuancée. Pourquoi? Parce qu'à l'heure du monde globalisé, il est désormais connu que la littérature contemporaine au Québec ne privilégie plus les discours identitaires fermés sur eux-mêmes ni la qualification d'être de «souche»; cette littérature examine des perspectives multiculturelles et transculturelles, elle «se déplace dans le contexte de la légitimation des déplacements géographiques et symboliques. [...] elle tend à explorer les facettes des diversités planétaires» (IMBERT, 2013, p. 9). Ou encore, comme le souligne Patrick Imbert, dans cette littérature, «on cherche à comprendre les valeurs différentes et voir comment peut s'établir un vivre-

\footnotetext{
${ }^{4}$ Rappelons l'allocution prononcée par Gaston Miron lors de la remise du Prix Athanase-David, en octobre 1982, qui faisait l'apologie de l'État québécois et d'une identité québécoise forte et «différente» du reste du Canada: "Le phénomène de la Révolution tranquille entre en conflit avec le fédéralisme dans lequel nous vivions. C'est la dimension politique de la culture - vécue comme différence depuis 1945 - qui fait irruption et nous fait accéder à la conscience d'être un tout. Les signes de l'identité effectuent leur dernière mutation: nous nous proclamons des Québécois. Vers 1963, nous parlons de l'État québécois, de la littérature québécoise, du cinéma québécois, de l'économie québécoise... Et quand on se conçoit comme un tout, on veut avoir les pouvoirs du tout». ("Les signes de l'identité», Québec français 52, 1983, p. 22).
} 
ensemble efficace émotionnellement, économiquement et intellectuellement» (2013, p. 10). On voit ainsi que l'important est de saisir des passerelles, des liens avec les autres, d'entamer des rencontres, plutôt que de mettre de l'avant un discours d'identité-singularité qui exclut dès qu'on prononce «nous autres» vs. "vous autres», «eux autres». Dans cette perspective, le titre de l'essai de Régine Robin Nous autres, les autres, paru en 2013, montre justement qu'il est possible de disloquer, déplacer, détourner le dualisme: «nous autres, les autres» subvertit l'idée de nationalisme québécois et nous conduit à changer l'angle de vue, à poser le regard sur le groupe des autres - les immigrants, par exemple - pas d'emblée sur le groupe des Québécois de naissance.

Publié en 1983, La Québécoite de Robin a connu un succès considérable et constitue aujourd'hui un incontournable dans l'analyse du phénomène de «l'écriture migrante» et des questions que pose la nouvelle posture de l'écrivain québécois depuis les années $1980^{5}$. Or, il est intéressant de noter que dans La Québécoite, la narratrice utilise le mot "autre» pour faire part de sa solitude et de sa marginalité: «Quelle angoisse certains aprèsmidis - Québécité, québécitude - je suis autre. Je n'appartiens pas à ce Nous si fréquemment utilisé ici - Nous autres - Vous autres» (ROBIN, 1983, p. 53-54). Rejetée d'une identité communautaire qu'elle ne peut et ne veut pas assimiler, elle erre dans son espace à elle qui n'est ni ici ni là-bas. C'est un territoire dont les frontières ont éclaté sous le choc de l'exil et qui est désormais impossible à délimiter. La narratrice et le personnage du récit cherchent en vain ce qui a été perdu: l'identité d'avant, un sentiment d'appartenance, quelque chose qui serait possible à cerner et qui mettrait fin à l'errance. Ainsi, l'espace de $\mathrm{La}$ Québecoite ne se forge pas directement au croisement des deux identités, mais surgit de la recherche, de l'oscillation constante entre ces deux identités. Ce territoire engendré par le mouvement de va-et-vient est ce qu'on désigne, selon les mots de Robin, par «l'entre-deux». C'est par cet entre-deux en quête d'un sentiment d'appartenance que se raconte l'expérience migrante. Des critiques (Simon Harel) ont souligné la prégnance de l'entre-deux dans le roman, sans pour autant penser l'éclatement des espaces topique, langagier et

\footnotetext{
${ }^{5}$ Voir: Fulvio Caccia (dir.), La transculture et ViceVersa, Montréal, Tryptique, 2010, et Susanne Gehrmann et Claudia Gronemann (dir.), Les enJEux de l'autobiographie dans les littératures de langue française, Paris, L'Harmattan, 2006.
} 
mémoriel pour rendre compte de l'étrangeté de la solitude qui est le lot de l'exilée. Et c'est justement de juive errante qu'on pourrait qualifier la Québécoite qui, en se déplaçant constamment, crée un territoire qui lui est propre, dans les plis de l'entre-deux; dans l'espoir d'appartenir.

Pourtant, que signifie "appartenir»? C'est une interrogation qui revient sous une forme ou une autre à travers toute l'œuvre philosophique de Jacques Derrida. On y entend aussi bien la volonté d'interroger les crispations identitaires actuelles que la remise en cause de toute idée d'appartenance, qu'elle soit nationale, linguistique, communautaire ou sexuelle. Chez Derrida, la question est au moins double: premièrement, que reste-t-il de nos appartenances, de l'héritage commun, de ce qui, d'une certaine façon et selon des voies multiples, nous fait tenir ensemble ? Question nostalgique mais qui, plus largement, recouvre celle du sens partagé de la communauté. Deuxièmement: comment réinventer des nouvelles formes d'appartenance, sous quelles conditions, dans quelles limites? Question qui concerne l'à venir, comme disait Derrida, et là encore, plus largement le sens à donner à toute œuvre - littéraire ou philosophique - œuvre d'art ou œuvre de vie. D'une part, le refus de l'appartenance au sens de l'identité, de l'enracinement, du propre, du soi-même comme un; et d'autre part, la nécessité de se forger une appartenance par la créativité et la création artistique et de pensée. Dans cet entre-deux, on reconnaît également les questionnements de Régine Robin dans la Québécoite, mais aussi au fil de son œuvre. Chez Robin et chez Derrida, l'appartenance tient conjointement de l'impossible et du nécessaire. La question n'est pas simple, elle est autant théorique qu'intimement douloureuse. On se souvient de la belle formule de Circonfession: «qui suis-je si je ne suis pas ce que j'habite et où j'ai lieu?» (DERRIDA, 1991, p. 32).

Rappelons que dans La Québécoite, l'incipit devient un leitmotiv unificateur: il ne rend pas compte seulement de l'espace urbain et de la structure du récit, mais également de la mémoire désarticulée: «Pas de lieu, pas d'ordre /Mémoire divisée à la jointure des mots /Les couches muettes du langage, brisées/ La parole immigrante en suspens / entre / deux HISTOIRES» (ROBIN, 1983, p. 152). La rupture de la linéarité est évidente. Une fois divisée, la mémoire ne peut plus être unifiée. De cette manière, elle fait surgir l'entredeux mémoriel de la grande Histoire, du passé dont la narratrice ne parvient pas à se détacher dans le présent. Le passé européen persiste à faire irruption 
dans l'espace du Nouveau Monde, comme pour rappeler la rupture, mais également l'horreur, l'impossible:

Il n'y a pas de métaphore pour signifier Auschwitz pas de genre, pas d'écriture. Écrire postule quelque part une cohérence, une continuité, un plein du sens [...]. Rien qui puisse dire l'horreur et l'impossibilité de vivre après. Le lieu entre le langage et l'Histoire s'est rompu. Les mots manquent. Le langage n'a plus d'origine ni de direction. Quel temps employer ? Il n'y a qu'un présent éternel. Un présent qui ne passe pas. Le poids de ces millions de morts m'étouffe. En errance d'Europe en Amérique avec ces morts encombrants qui réclament leur dû dans un silence assourdissant (ROBIN, 1983, p. 141).

Finalement, la mémoire d'un passé obsédant - parce qu'impossible à oublier - force le déplacement, l'errance qu'elle soit spatiale ou langagière. Aux prises avec l'errance, la narratrice et le personnage trouvent dans l'exil un espace qui ne peut pas être véritablement nommé, mais qui est un espace tout de même: celui de l'entre-deux. Femme étrangère, autre, en exil à Montréal, femme autre à Paris, la Québécoite - tout comme l'écrivaine Régine Robin - se définit par le mouvement entre cette ville-ci et cette ville-là, entre un récit et un autre, entre le passé et le présent. Elle erre dans l'entre-deux ouvert sur le multiple; elle emprunte des voies sinueuses, pas de chemin en ligne droite. D'ailleurs, la narratrice se déplace en oscillant, en s'approchant de ceci pour s'éloigner de cela, puis s'approchant de cela pour s'éloigner de ceci. Par ces itinéraires de vie et d'écriture, dans le mouvement du désordre intérieur et extérieur, une voix se fait entendre, voix incertaine et fragile, qui hésite et parfois perd son souffle. Mais cette voix résonne malgré tout, ébranle les certitudes, parvient à inscrire... l'expérience de l'immigrante, «les mots de l'autre-espace. Ils n'ont plus de place» (ROBIN, 1983, p. 86).

La Québécoite reprend constamment un topos que l'on pourrait qualifier de superposition d'existences. Dans le récit, tout se superpose, s'ajoute à ce qui le précède. Ainsi, les espaces s'accumulent, les histoires s'additionnent les unes aux autres. Il en va de même pour la mémoire où s'enchevêtrent passé, présent et désir d'avenir dans une temporalité éclatée, qui rompt la chronologie. Dans ce récit, l'existence se vit comme une continuité dans le désordre créatif. L'éclatement temporel est étroitement lié à l'éclatement mémoriel, puisque certains souvenirs en viennent à se greffer au présent morcelé et instable associé à l'immigration. Ces intermittences appellent à la fois l'éclatement spatial puisque le présent est vécu à Montréal, 
dans cette ville où surgissent les souvenirs vécus ailleurs: «Montréal semble permettre la sédimentation des mémoires, elle qui se caractérise par sa porosité alors que Paris tient lieu de centre métropolitain relativement immuable, elle est la ville de l'enfance et des années de formation. [...]. Ville centrale dans La Québécoite de Régine Robin, mais qui est toujours perçue à travers le prisme montréalais» (1994, p. 164), rappelle Simon Harel.

Après tout, vivre dans une de ces villes nécessite le souvenir de l'autre ville, celle laissée derrière; les souvenirs se superposent comme les espaces auxquels ils sont associés, multipliant l'identité de l'immigrante. Paris et Montréal sont ainsi étroitement liées malgré la place excessivement différente que ces villes occupent dans la mémoire de la narratrice et du personnage qui glissent d'une ville à une autre, d'une histoire à une autre, d'une culture à une autre:

Il y avait les CANONS/le CANON de TOLBIAC /et /les BOUQUET DE L'OPERA/LE BOUQUET DE VERSAILLES/LE BOUQUET DE L'OPÉRA (...) / sans compter LES CHIENS/ PENDANT LA GUERRE.

Comme le ratage de l'exil même. [...] Un express bien tassé - bon - je me taille. Je me calte. Je lève l'ancre. Je fiche le camp. Je me tire. Je mets les bouts. Je mets les voiles. La voile. "Après une navigation de trois mois, ils arrivaient. C'était un trajet dangereux. (...) Vous paierez 6000 livres dit l'ordre royal du 12 mars 1534 au pilote Jacques Cartier qui va aux Terres Neuves découvrir certaines îles et pays où l'on dit qu'il se doit trouver grande quantité d'or» (ROBIN, 1983, p. 22).

De fait, la narratrice dit de multiples façons son désir de partir, mais où et vers quoi? Impossible de le dire puisqu'elle passe d'un souvenir à un autre, d'une histoire qui lui est personnelle à d'autres qui sont, elles, collectives. Ce désir de s'en aller la mène de Paris à Montréal, de Montréal à Paris, inter-changeant sans cesse la destination et le lieu de départ. En faisant allusion à la grande Histoire, à l'occupation nazie en France et à la découverte du Canada, la narratrice passe d'un souvenir - ici, l'occupation nazie - qui la constitue comme sujet immigrant à un souvenir associé à une histoire qui lui restera toujours étrangère - celle de la fondation du Canada. Il y a un brouillage mémoriel qui s'engendre dans ce passage d'une remémoration à une autre, lorsque passé et présent se confondent, entraînant dans ce mouvement déstabilisant, quelque chose comme le ratage de l'expérience de l'exil. Car, celle qui n'est pas à même de se souvenir d'où elle vient et où elle va 
est certainement en exil, mais un exil sans référent, précaire, qui correspondrait à l'impossibilité de l'unité (même fragile) du sujet immigrant à jamais morcelé. D’ailleurs, au niveau du récit, l'unité devient inconcevable, comme l'éclatement de la ville et la langue multiple l'ont révélé. Simon Harel souligne avec justesse: "La structure du roman témoigne d'un reconquête identitaire impossible» (1992, p. 409). Il en va de même pour la remémoration qui, plutôt que d'aider la femme immigrante à se rappeler qui elle est, ne cesse de la défaire, de la fragmenter. L'identité est morcelée par la mémoire.

\section{Conclusion}

«La grande saveur des frontières, une fois reconnues et garanties, c'est qu'on peut les franchir, jouer à leurs marges, exercice autrement plus exaltant que leur abolition pure et simple. Seuls les conquérants rêvent d'effacer les frontières, surtout celles des autres» (2011, p. 346), écrit Régine Robin dans son essai Nous autres, les autres: difficile pluralisme. En fait, aucune écriture ne peut échapper aujourd'hui à la traversée des frontières, à la pluralisation, au dialogisme, aux dispositifs les plus variés du déracinement, donc aux transgressions thématiques, linguistiques, symboliques et culturelles. Précisément, comme le dit le manifeste «Pour une littérature monde en français» paru dans le journal Le Monde en 2007, les configurations du champ littéraire francophone, la production littéraire de langue française et la réception se sont mises à bouger. Le contenu de ce manifeste prend acte des changements et cherche à mettre fin aux dominations, aux distinctions entre centre et périphéries, en proclamant la fin de la francophonie et l'avènement d'une littérature aux frontières ouvertes, où pour toute une nouvelle génération d'écrivains, la multiplicité des langues et des cultures va de soi.

Au Québec, l'établissement du corpus d'auteurs nés à l'étranger et l'étude des déterminations migratoires, sociales, économiques qui le parcourent permettent de mieux comprendre l'histoire de la vie littéraire québécoise grâce à l'ampleur de cet apport. Le phénomène littéraire des écritures dites de la migration apparaît comme un courant littéraire fascinant notamment parce qu'il lie, de manière historique, l'évolution de la littérature québécoise aux grands courants de pensée de la fin du $20^{\mathrm{e}}$ siècle, défini par le postmodernisme et la migrance, par le transculturalisme et la reconnaissance 
des altérités, mais aussi à une certaine condition identitaire qui tend aujourd'hui à la multiplicité des appartenances, à la transmission d'héritages pluriels. Au Québec donc, véritable laboratoire de ces nouvelles formes qui s'expérimentent: postnational, transnational, transculturel, métissage, créolisation, migrance..., on assiste à la mobilité d'une écriture de réajustement des temps présents, où il est possible «d'aller de surprise en surprise, comme dans un rêve, et c'est ce que devrait être la vie» (2015, p. 23), comme dit Dany Laferrière. Ces sont les écritures trans-migrantes qui façonnent le nouvel imaginaire, et qui confèrent une nouvelle dimension aux littératures nationales ouvertes désormais à une relecture des marges et des frontières. C'est du moins le pari qui se fait dans la transculturalité du monde qui est le nôtre.

\section{Referências}

BOUCHARD, Gérard. L'Interculturalisme: un point de vue québécois, Montréal: Boréal, 2012.

CACCIA, Fulvio (dir). La transculture et ViceVersa, Montréal: Tryptique, 2010.

DERRIDA, Jacques. "Circonfessions», in: Jacques Derrida et Geoffrey Bennington (dir.), Jacques Derrida, Paris: Seuil, 1991, p. 26-52.

DES ROSIERS, Joël. Théories caraïbes, Montréal: Tryptique, 1996.

DUPONT, Éric. La logeuse, Montréal: Marchand de feuilles, 2006.

GEHRMANN, Susanne et Claudia GRONEMAN (dir.). Les enJEux de l'autobiographie dans les littératures de langue française, Paris: L'Harmattan, 2006

HAREL, Simon. «La parole orpheline de l'écrivain migrant», in: Pierre Nepveu et Gilles Marcotte (dir.), Montréal imaginaire. Ville et littérature, Montréal: Fides, 1992, p. 373-418.

HAREL, Simon. «Montréal, une 'parole' abandonnée. Gérard Étienne et Régine Robin", dans Benoît Melançon et Pierre Popovic (dir.), Montréal 16421992. Le Grand passage, Montréal, XYZ, 1994, p. 154-172.

IMBERT, Patrick. Comparer le Canada et les Amériques. Des racines aux réseaux, Québec: Presses de l'Université Laval, 2014. 
IMBERT, Patrick (dir.). Rencontres multiculturelles. Imprévus et coïncidences. Le Canada et les Amériques, Ottawa: Chaire de recherche "Canada: Enjeux sociaux et culturels dans une société du savoir», 2013.

KOKIS, Sergio. Le Pavillon des miroirs, Montréal: Lévesque éditeur, 1994.

KYMLICKA, Will. Multicultural Odysseys: Navigating the New International Politics of Diversity, Oxford: Oxford University Press, 2009.

LAFERRIÈRE, Dany. Dany Laferrière à l'Académie française. Discours de réception. Réponse d'Amin Maalouf, Montréal: Boréal, 2015.

OUELLET, Pierre. Où suis-je? Paroles des Égarés, Montréal: VLB Éditeur, 2010.

OUELLET, Pierre. L'esprit migrateur. Essai sur le non-sens commun, Montréal: VLB Éditeur, 2005.

ROBIN, Régine. "Écrire français avec un accent », in: Fabula-LhT, n 12, «La langue française n'est pas la langue française», mai 2014, en ligne: http://www.fabula.org/lht/12/robin.html (page consultée le 4 juillet 2017).

ROBIN, Régin. Nous autres, les autres: difficile pluralisme, Montréal: Boréal, 2011.

ROBIN, Régine. La Québécoite, Montréal, Québec/Amérique, 1983. Prix Jacques-Rousseau. Prix du Jewish Book Award pour la version anglaise The Wanderer. Réédition Montréal, Typo, 1993, avec une postface de l'auteure, «De nouveaux jardins aux sentiers qui bifurquent», p. 207-224.

SIMON, Sherry. "Hybridités culturelles, hybridités textuelles», in: François Laplantine et al. (dir.), Récit et connaissance, Lyon: Presses de l'Université de Lyon, 1998, p. 233-234.

Recebido em 30 de abril de 2018.

Aceito em 28 de maio de 2018. 